Núñez Medina, G., Jiménez Acevedo, H.M. (2021). Análisis espacial de la fecundidad adolescente y programas sociales en Chiapas. GeoFocus (Artículos), Revista Internacional de Ciencia y Tecnología de la Información Geográfica, 27, 115-134. http://dx.doi.org/10.21138/GF.557

\title{
ANÁLISIS ESPACIAL DE LA FECUNDIDAD ADOLESCENTE Y PROGRAMAS SOCIALES EN CHIAPAS
}

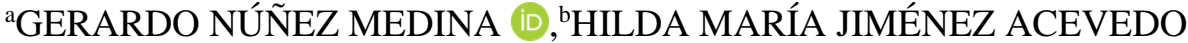 \\ Universidad Autónoma de Chiapas (UNACH) \\ Boulevard Belisario Domínguez, Km. 1081, Sin Número, 29050. Terán, Tuxtla Gutiérrez, \\ Chiapas, México \\ agerardo.nm1@gmail.com, b $\underline{\text { luzhec10@ hotmail.com }}$
}

\begin{abstract}
RESUMEN
El trabajo busca analizar el efecto espacial que los programas de apoyo gubernamental han ejercido sobre los niveles de fecundidad adolescente en los municipios de Chiapas al año 2015. Para cumplir con dicho objetivo, se planteó una estrategia basada en la aplicación de diferentes modelos de regresión espacial, que busca cuantificar el efecto de variables como la educación media femenina, el ingreso familiar laboral, el acceso a servicios médicos y los apoyos gubernamentales sobre la fecundidad adolescente observada en los municipios de Chiapas. Se encontró que el ingreso laboral no ejerce un efecto espacial significativo sobre la fecundidad adolescente, contrario a los ingresos recibidos a través de subsidios gubernamentales cuyo efecto estimado ha sido el de incrementar los niveles de fecundidad adolescente. Esta situación resulta paradójica, ya que se trata de recursos destinados a romper los ciclos de pobreza intergeneracional.
\end{abstract}

Palabras clave: heterogeneidad espacial; modelo espacial autorregresivo (SAR); modelo de errores espaciales (SEM); estimación directa de tasas de fecundidad.

\section{SPATIAL ANALYSIS OF ADOLESCENT FERTILITY AND SOCIAL PROGRAMS IN CHIAPAS}

\section{ABSTRACT}

This study seeks to analyze spatial effects that governmental support programs have had on adolescent fertility levels in the municipalities of Chiapas in 2015. To meet this objective, a strategy based on the application of different spatial regression models was proposed, which seeks to quantify the effect of variables such as female secondary education, family labor income, access to medical services and governmental support on adolescent fertility observed in 
Núñez Medina, G., Jiménez Acevedo, H.M. (2021). Análisis espacial de la fecundidad adolescente y programas sociales en Chiapas. GeoFocus (Artículos), Revista Internacional de Ciencia y Tecnología de la Información Geográfica, 27, 115-134. http://dx.doi.org/10.21138/GF.557

the municipalities of Chiapas. It was found that labor income does not exert a significant spatial effect on adolescent fertility, contrary to income received through government subsidies, whose estimated effect has been to increase adolescent fertility levels. This situation is paradoxical, since these resources are intended to break the cycles of intergenerational poverty.

Keywords: spatial heterogeneity; spatial autoregressive model (SAR); spatial error model (SEM); direct estimation of fertility rates.

\section{Introducción}

El presente trabajo tiene por objeto analizar el efecto que los programas sociales han ejercido sobre el comportamiento de la distribución espacial de la fecundidad adolescente, en los 121 municipios que integran el estado de Chiapas, México, al año 2015. Para lograr este objetivo, se utilizan una serie de modelos de regresión espacial que analizan la relación que existe entre la distribución espacial de la fecundidad adolescente y los niveles observados de educación, ingreso medio, acceso a programas sociales y acceso a servicios de salud. El objetivo es probar la hipótesis relativa a la existencia de un patrón de distribución espacial de formación de conglomerados, que concentran altos niveles de fecundidad adolescente en municipios con altos niveles de gasto social.

Es importante señalar que, si bien la adolescencia ${ }^{1}$ es considerada como una tapa muy compleja en el desarrollo del ser humano, que permite llegar a la madurez física, mental y emocional; las adolescentes no reúnen las condiciones óptimas para enfrentar la maternidad con plenitud y equilibrio; ya que las niñas y/o adolescentes no tienen la capacidad económica, ni la madurez emocional para procrear y cuidar a un bebé, además de los riesgos físicos inherentes (Welti, 2000: 45). Algunas adolescentes inician a edad temprana (12-19 años) una vida sexual activa, periodo en el cual sus decisiones ${ }^{2}$ estarán influenciadas por aspectos culturales, sociales, familiares, económicos y educativos, así como por el acceso a información, orientación y educación en materia de salud reproductiva, por lo que cada uno de estos aspectos se espera que presente una distribución espacial diferenciada.

Por ejemplo, la tasa de fecundidad adolescente (15-19) alcanzó un nivel de 48.9 por mil a nivel mundial, en los países en desarrollo alcanzó un nivel de 52.7, y específicamente, en América Latina y el Caribe llegó a los 73.2. Esta cifra fue sólo superior en África, donde alcanzó 103 por mil. Europa presentó el nivel más bajo con 19 por mil; todas las cifras para el periodo 20052010, publicadas por la división de población del Departamento de Asuntos Económicos y Sociales de las Naciones Unidas (Naciones Unidas, 2003).

Si bien a nivel global, la tendencia de la fecundidad adolescente durante la última década ha sido errática, su concentración espacial es clara a la par que la importancia del fenómeno

\footnotetext{
${ }^{1}$ La adolescencia en definición de la Organización Mundial de la Salud (OMS), es un "período de la vida en el cual el individuo adquiere la capacidad reproductiva, transita los patrones psicológicos de la niñez a la adultez y consolida la independencia socio-económica" y se trata del grupo de edad que va de los 10 a los 19 años de edad (Issler 2013:12). En México, la Ley para la Protección de los Derechos de Niñas, Niños y Adolescentes (LPDNNA) considera como adolescentes a las personas que tienen entre 12 años cumplidos y 18 años incumplidos, lo que deja fuera al grupo de 10 a 11 años (LPDNNA 2000).

${ }^{2} \mathrm{Al}$ no tener la madurez mental y emocional para hacer frente a la reproducción y maternidad. En el caso de menores de 12 años se considera un asunto de violencia o abuso de menores, que no está suficientemente atendido y visibilizado por la Ley en México.
} 


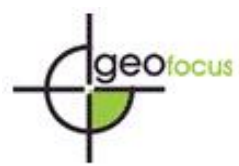

Núñez Medina, G., Jiménez Acevedo, H.M. (2021). Análisis espacial de la fecundidad adolescente y programas sociales en Chiapas. GeoFocus, Revista Internacional de Ciencia y Tecnología de la Información Geográfica, 27, 115-134. http://dx.doi.org/10.21138/GF.557

persiste. Entre el 15 y el 20 por ciento de los nacimientos a nivel global corresponden a madres adolescentes, y esta proporción se ha mantenido estable durante la última década. Hay indicios, al menos en Brasil y en Paraguay, de que la estructura de edad de las madres adolescentes se ha rejuvenecido en la última década, y esta situación parece confirmarse para el caso de Chiapas.

Resulta relevante que Chiapas sea la entidad con la tasa global de fecundidad más alta de México, al tiempo que su tasa de fecundidad adolescente sea también la más alta del país; lo que históricamente coincide con ser la entidad federativa que concentra el mayor gasto social desde el inicio de los programas en 1990. Por ello, una de las preocupaciones centrales del trabajo es la de analizar la posible relación que existe entre la aplicación de los programas sociales y el nivel observado de la fecundidad adolescente en los municipios de la entidad, donde el diseño de los programas pudiera involuntariamente estar modificando el comportamiento reproductivo de las adolescentes, especialmente en los municipios de mayor influencia de los programas.

Adicionalmente debe considerase el hecho de que un porcentaje importante de embarazos en adolescentes es producto del abuso y/o violencia sexual, causa que puede ser atribuida a embarazos en menores de 12 años. Deben reconocerse, además, las complejas condiciones asociadas con el embarazo adolescente y la maternidad temprana, donde convergen el contexto macro, el contexto social local y el entorno familiar, que contribuyen a definir las opciones efectivas disponibles para que las adolescentes sean capaces de elegir su plan de vida (Hernández et al., 2015, Azevedo et al., 2012, BM 2012).

\section{Antecedentes}

Desde el año 2012, el tema del embarazo en adolescentes ha venido ocupando un espacio importante en la agenda pública de México. No solo por los aspectos de salud sino por sus múltiples implicaciones sociales (PROMAJOVEN 2012). El embarazo en adolescentes se ubica en una dimensión socio-cultural que involucra a los sectores: educativo, de salud, económico, familiar y penal.

El riesgo de tener un embarazo adolescente está determinado por aspectos relacionados con: el inicio temprano de una vida sexual activa, el conocimiento y uso de métodos anticonceptivos, la condición económica (oportunidades de empleo) y la condición social (educación, salud, residencia, cultura), entre otros. En este sentido, existe evidencia de que la difusión de información sobre el acceso y uso de métodos anticonceptivos no necesariamente reduce los embarazos tempranos, al menos de forma significativa, dado que la maternidad para algunas adolescentes se presenta como una meta, la cual es determinada por distintos factores sociales, por ejemplo: el matrimonio (o la vida en pareja) resulta una opción de vida y/o superveniencia viable, frente a la falta de oportunidades de desarrollo (Welti s/f, Welti, 2000:46, Ehrenfeld, 2011:19).

Adicionalmente, el nivel de uso de métodos anticonceptivos está ligado no sólo a la información disponible y a su acceso, sino que además deben sortearse barreras ligadas con la capacidad de negociación de las adolescentes frente a sus parejas, lo que en muchas ocasiones resulta en un claro impedimento para su uso (Ehrenfeld, 2011:20). Por lo cual, las adolescentes mayormente expuestas a este tipo de situaciones son aquellas que presentan menores grados de empoderamiento y bajo nivel educativo.

Se ha discutido ampliamente si el embarazo entre jóvenes estudiantes las expulsa de la escuela, o si las mujeres que se embarazan en la adolescencia son aquellas que de cualquier manera están limitadas para continuar estudiando por falta de recursos. En México, el embarazo adolescente 
Núñez Medina, G., Jiménez Acevedo, H.M. (2021). Análisis espacial de la fecundidad adolescente y programas sociales en Chiapas. GeoFocus, Revista Internacional de Ciencia y Tecnología de la Información Geográfica, 27, 115-134. http://dx.doi.org/10.21138/GF.557

representa la cuarta causa de deserción escolar entre mujeres de 15 a 19 años, pero también antes de los 15 años existen alrededor de 7 mil nacimientos de madres niñas (Welti, 2000). El hecho es que la fecundidad adolescente es más frecuente en contextos de pobreza donde las limitaciones económicas familiares hacen pensar que: "invertir en la educación de las mujeres tiene bajas expectativas de retorno" (Azevedo et al., 2012:29, Stern y Menkes, 2008: 45). Por otra parte, un mayor tiempo dedicado a estudiar tiene efectos en la disminución de los embarazos adolescentes, de forma que mujeres con un mayor nivel de educación incrementan su capacidad para tomar mejores decisiones en cuanto a su reproducción y sexualidad (Berthelon y Kruger, 2011, Cortés et al., 2011).

Desde el punto de vista de la teoría de la transición demográfica, la evolución de la fecundidad en México y Latinoamérica puede dividirse en tres grandes etapas: (1) pretransicional de 1950 a 1970, caracterizada por el inicio en la alteración de las tasas de fecundidad que hasta antes del inicio del periodo habían permanecido altas y estables; (2) periodo transicional de 1970 a 1990, donde se observa una reducción acelerada de la fecundidad en todos los grupos de edad; y (3) etapa postransicional de 1990 a 2015, definida por un proceso de baja reducción de las tasas de fecundidad en el que se espera se estabilicen en niveles cercanos al reemplazo intergeneracional. Tanto en México como en Latinoamérica se observaron tasas globales de fecundidad superiores a los 6 hijos promedio por mujer, durante la primera etapa y a finales de la tercera etapa, el promedio de hijos por mujer fluctuaba alrededor de los 2.1 hijos.

Si bien, se habla de una reducción generalizada de los niveles de fecundidad en todos los grupos de edad, la reducción ocurrió de manera heterogénea con grandes diferencias entre países de la región y entre grupos de edad. Diferentes estudios (Flórez et al., 2004, Ordóñez y Murad, 2002) señalan que la maternidad entre adolescentes descendió a un ritmo mucho menor que la fecundidad general, situación que se agudizó especialmente entre mujeres en condiciones socioeconómicas desfavorables o que habitan en áreas rurales o dispersas. Es decir, distintos factores socioeconómicos han tenido la capacidad de modificar el comportamiento de la fecundidad adolescente, en especial, destacan factores como el nivel educativo, el ingreso y el acceso a servicios de salud.

En el mismo sentido, se ha documentado la existencia de una relación inversa entre los niveles de fecundidad adolescente y el ingreso familiar, donde las adolescentes que viven bajo condiciones de pobreza o pobreza extrema tienen una mayor probabilidad de convertirse en madres, respecto de las adolescentes que viven con niveles de ingreso superiores a la línea de pobreza. La fecundidad adolescente en áreas rurales de Latinoamérica es en promedio entre 1.5 y 2.5 veces mayor que la registrada en zonas urbanas, mientras que la tasa de fecundidad registrada en adolescentes con niveles de escolaridad inferiores a primaria es tres veces mayor en relación con adolescentes de nivel educativo equivalente al bachillerato o superior. Sin embargo, la relación causal entre la permanencia en el sistema educativo y la fecundidad en adolescentes no es clara, en el sentido de si la deserción escolar temprana incrementa la fecundidad adolescente, o si el embarazo adolescente incrementa los niveles de deserción escolar precoz y en qué grado se encuentra asociado con el ingreso.

El efecto de la aplicación de programas sociales sugiere que a medida que mejoran las condiciones económicas de los hogares, es de esperar una disminución de la fecundidad adolescente (Guzmán et al., 2000), debido a que el efecto de la pobreza sobre la tasa de fecundidad adolescente se transmite a través del nivel educativo, donde la interacción entre los niveles de ingreso y la escolarización ejercen efectos conjuntos sobre la fecundidad en adolescentes. Lo cual puede entenderse dado que, en estratos económicos bajos, los altos niveles de fecundidad operan como un factor que busca garantizar la subsistencia en el largo plazo de los padres, lo que evidentemente termina formando un ciclo perverso, donde la persistencia de 


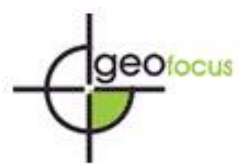

Núñez Medina, G., Jiménez Acevedo, H.M. (2021). Análisis espacial de la fecundidad adolescente y programas sociales en Chiapas. GeoFocus, Revista Internacional de Ciencia y Tecnología de la Información Geográfica, 27, 115-134. http://dx.doi.org/10.21138/GF.557

unión-maternidad temprana se encuentra incrustada en una estructura sociocultural arraigada en contextos rurales que legitima socialmente dicho comportamiento reproductivo, o donde la maternidad adolescente que se presenta en ausencia de una unión conyugal (sin emancipación familiar) pudiera estar asociada a contextos de violencia.

Una de las hipótesis que se sostiene en esta investigación es que la relación entre una mayor incidencia de fecundidad adolescente en poblaciones pobres está ligada a la aplicación de programas sociales, donde las jóvenes, sus familias y sus parejas consideran atractivo incorporarse a los programas de trasferencias condicionadas, vía el embarazo temprano. Según Azevedo et al. (2012), las mujeres mexicanas que dieron a luz durante la adolescencia en 2011, presentan mayor propensión a participar en los programas sociales y una mayor dependencia de los ingresos de la asistencia social. Esto significa que, una vez que las adolescentes descuentan el costo personal de la maternidad, los beneficios futuros esperados de una maternidad temprana, incluidos los programas sociales, les resultan favorables.

Según la Encuesta Nacional de Salud 2012, en México del total de las mujeres adolescentes de 12 a 19 años de edad que tuvieron relaciones sexuales, el $51.9 \%$ alguna vez había estado embarazada y $10.7 \%$ estaba embarazada al momento de la entrevista. El número de nacimientos por cada mil mujeres fue de 30.0, superior a la tasa de fecundidad observada en 2005 y de las mujeres de 12 a 19 años de edad, que fue de 37.0 nacimientos por cada mil mujeres. También la encuesta reporta que $10.3 \%$ de las mujeres había sufrido agresión sexual. El $46.0 \%$ de las agresiones se presentaron en la vía pública, $29.8 \%$ en la escuela y el $11.3 \%$ en el hogar (ENSANUT 2012:19). Valencia y Schiavon (2014) encontraron que entre 2008 y 2011 se certificaron 8 millones 263 mil 020 nacimientos, de los cuales $0.7 \%$ fueron en adolescentes muy jóvenes de 10-14 años y $18.9 \%$ fueron en adolescentes entre 15 y 19 años (Valencia y Schiavon, 2014:19). Por su parte, la Estrategia Nacional para la Prevención del Embarazo Adolescente señala que el promedio nacional de nacimientos de las mujeres de 15 a 19 años fue de 69.5 nacimientos por cada mil mujeres; Chiapas tuvo una tasa mayor a 75 por mil (ENADID 2016:26). El INEGI señala que las adolescentes indígenas tienen una tasa de fecundidad más alta que las no indígenas, de las mujeres de 15 a 19 años hablantes de una lengua indígena la tasa era de 96.7 por mil mujeres y las no indígenas de 68 por mil (INEGI 2010). Los nacimientos en mujeres menores de 15 años se ubican en municipios más marginados que los de mujeres entre los 15 y los 19 años.

En el caso de Chiapas, la problemática del embarazo en adolescentes se enmarca en una serie de determinantes asociados a la marginación, el analfabetismo y los niveles de escolaridad, según el Consejo de Nacional Población (CONAPO 2010). Respecto de la salud reproductiva en adolescentes, la Encuesta Nacional de Salud y Nutrición 2012 señala que, en Chiapas el $70.2 \%$ de los menores entre 12 y 15 años indicaron haber escuchado de algún método anticonceptivo y el $78.3 \%$ del grupo de 16 a 19 años. En lo referente al embarazo en adolescentes en Chiapas, los resultados muestran que $73.0 \%$ del total de las mujeres de entre 12 y 19 años que ha iniciado una vida sexual, alguna vez han estado embarazadas (ENSANUT 2012:20).

\section{Fuentes de datos y estimación de variables}

Los datos utilizados para la elaboración de este trabajo se obtuvieron de la Encuesta Intercensal 2015 levantada por el Instituto Nacional de Estadística y Geografía (INEGI), de donde se compiló información sobre fecundidad, escolaridad femenina municipal promedio, ingreso medio mensual familiar, proporción de mujeres inscritas al Seguro Popular y proporción de 


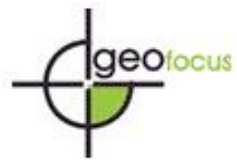

Núñez Medina, G., Jiménez Acevedo, H.M. (2021). Análisis espacial de la fecundidad adolescente y programas sociales en Chiapas. GeoFocus, Revista Internacional de Ciencia y Tecnología de la Información Geográfica, 27, 115-134. http://dx.doi.org/10.21138/GF.557

hogares que reciben algún apoyo a través de programas gubernamentales como: Prospera, Oportunidades, Adultos mayores y PROCAMPO, entre otros (INEGI 2015).

El Estado de Chiapas tiene una superficie de 73,311 kilómetros cuadrados lo que representa el $3.7 \%$ de la superficie total de México. La mayor parte de la superficie del Estado se conforma por las sierras de Chiapas y Guatemala y la cordillera Centroamericana. Al extremo Este se encuentra limitado por Guatemala, al Norte hace frontera con el estado de Tabasco, al Oeste se limita por los estados de Veracruz y Oaxaca, mientras que al Sur con el Golfo de Tehuantepec. Chiapas se encuentra constituido por 121 municipios y una población de 5.2 millones de habitantes al año 2015.

La Encuesta Intercensal 2015 garantiza representatividad a nivel municipal, en especial, en municipios aislados y con un alto porcentaje de localidades rurales dispersas en temas como: población total, estructura por edad y sexo, fecundidad, mortalidad, acceso a servicios de salud, educación, trabajo no remunerado y características económicas. Los datos de la Encuesta Intercensal 2015, permitieron estimar las tasas específicas de fecundidad (TEF) por edad, a nivel municipal para Chiapas. Las TEF municipales permitieron calcular la tasa de fecundidad adolescente considerando el grupo de edad de 10 a 19 años. Es importante señalar que, el procesamiento de datos, la estimación de tasas, índices y modelos espaciales se realizaron utilizando el lenguaje de programación $\mathrm{R}$ en su versión 3.2.2 y los paquetes gstat, spatstat y maptools (R Core Team 2013).

\section{1. Estimación de tasas de fecundidad adolescente}

La carencia de cifras sobre fecundidad adolescente a nivel municipal en Chiapas obligó a realizar una estimación de las mismas, para ello se decidió reconstruir la historia de la fecundidad general en Chiapas desde el año de 1970, utilizando la metodología de estimación propuesta por el UNFPA en "Direct estimation of fertility from survey data containing birth histories", disponible en la página (UNFPA, s/f). En la figura 1, se muestran las estimaciones de la tasa específica de fecundidad por año calendario.

Figura 1. Tasas globales de fecundidad Chiapas, 1970 a 2015

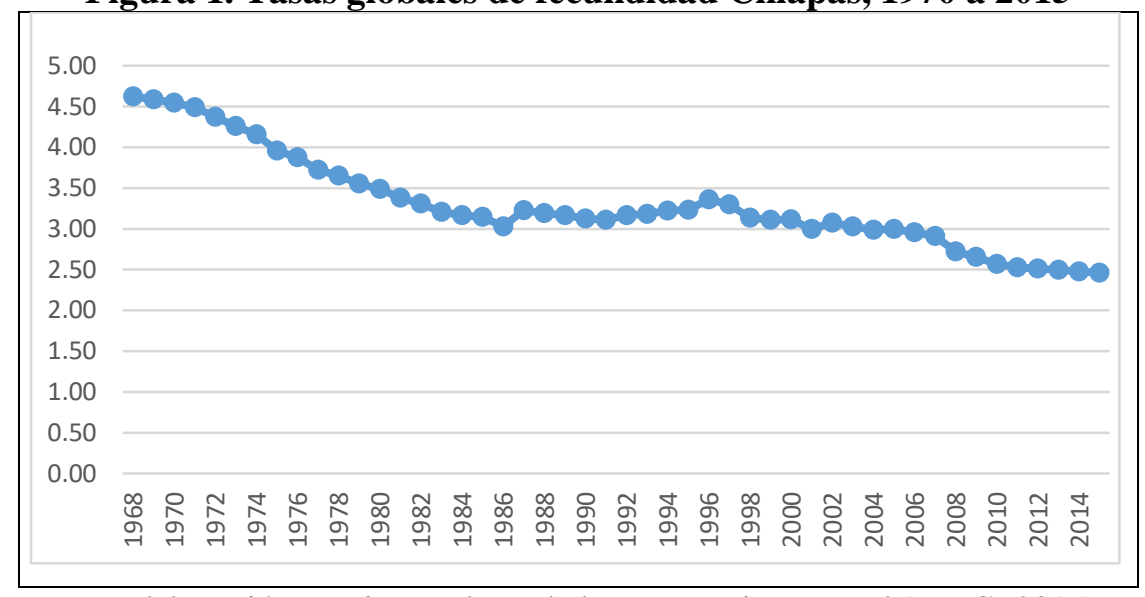

Fte.: Elaboración propia con datos de la encuesta intercensal (INEGI 2015).

Como se puede apreciar en la figura 1, el comportamiento de la fecundidad en la década de 1970 dibuja el inicio de una importante reducción de fecundidad desde un nivel medio superior a los 4.5 hijos por mujer. En las décadas de 1980 y 1990, se observa una importante reducción en los niveles de fecundidad general, sin embargo, debido a las recurrentes crisis económicas 
Núñez Medina, G., Jiménez Acevedo, H.M. (2021). Análisis espacial de la fecundidad adolescente y programas sociales en Chiapas. GeoFocus, Revista Internacional de Ciencia y Tecnología de la Información Geográfica, 27, 115-134. http://dx.doi.org/10.21138/GF.557

experimentadas por México durante esos años, el descenso de los niveles de fecundidad ocurrió a un ritmo mucho menor y con algunos retrocesos, adicionalmente se debe señalar que la fecundidad en adolescentes comienza a ser importante en esos años. Posterior al año 2000, se observa un estancamiento sostenido de los niveles de fecundidad, con un inicio más temprano de la etapa reproductiva que inicia desde los 15 años; la curva de fecundidad durante la década del 2000, alcanzó su nivel máximo alrededor de los 30 años; es importante notar que en este periodo se observó un importante crecimiento de la fecundidad adolescente, en especial, en mujeres menores de 17 años.

La suma de las tasas específicas de fecundidad entre los 10 y 19 años, en los diferentes municipios, define la tasa de fecundidad adolescente municipal, misma que será analizada con mayor detenimiento en el siguiente apartado.

\section{Metodología}

Los datos espaciales contienen información transversal localizada en coordenadas (latitud y longitud) con lo que se encuentran georreferenciadas (Elhorst, 2010); en este caso, se emplearon las tasas específicas de fecundidad adolescente de 10-19 años, escolaridad media, ingreso mensual y acceso a subsidios en los programas Prospera y Seguro Popular, referenciados a nivel municipal. Los componentes espaciales permiten modelar patrones de dependencia a través de: (1) la utilización de variables (dependientes o independientes) que capturan la dependencia espacial como un promedio ponderado de los valores vecinos, lo que se conoce como modelos de rezago espacial; o (2) utilizan un término de error, mediante la introducción de un proceso autorregresivo de error espacial, lo que se conoce como modelos de error espacial (Anselin, $1988 ; 1995)$.

El uso de métodos econométricos espaciales se basa en su capacidad para modelar variables en un contexto de regresión lineal, que permite cuantificar el impacto del espacio físico sobre el comportamiento de las variables estudiadas en datos de corte transversal. Es decir, la incorporación del concepto de dependencia espacial, que es el primer y más importante efecto de los modelos econométricos espaciales, se traduce en la integración de una relación funcional de las variables con el espacio, lo que resulta fundamental en caso de que el fenómeno demográfico, social o económico estudiado se encuentre, o se sospeche, que está espacialmente determinado (Fischer y Getis, 2010).

Antes de iniciar cualquier proceso de modelación espacial, es recomendable realizar un análisis exploratorio de datos espaciales con la finalidad de descubrir patrones de agrupamiento espacial, identificar estructuras o detectar valores aberrantes espaciales, por medio de estadísticos, diagramas, gráficas y/o mapas (Chasco, 2003).

\subsection{Análisis exploratorio de datos espaciales}

El análisis exploratorio de datos espaciales (AEDA) estudia la correlación entre una variable y el espacio geográfico sobre el que se distribuye, lo que se conoce como autocorrelación espacial. Tobler (1970) establece en su primera ley que: "todo está relacionado con todo, pero las cosas cercanas están más relacionadas que las cosas distantes", con lo que sienta las bases para definir el concepto de autocorrelación espacial como una medida de asociación entre unidades espaciales o regiones en función de un atributo. 


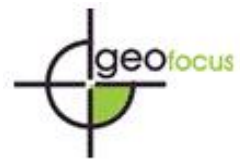

Núñez Medina, G., Jiménez Acevedo, H.M. (2021). Análisis espacial de la fecundidad adolescente y programas sociales en Chiapas. GeoFocus, Revista Internacional de Ciencia y Tecnología de la Información Geográfica, 27, 115-134. http://dx.doi.org/10.21138/GF.557

Durante la etapa inicial del AEDA es necesario definir la matriz de ponderadores espaciales, a partir de la identificación y ponderación de las relaciones de vecindad observadas en todas las unidades espaciales (municipios), para lo que existen diferentes opciones (Ripley 1981). En esta investigación se emplea el criterio de vecino más cercano, que define dos unidades espaciales como vecinas cuando están situadas dentro de una banda, definida como la distancia máxima entre centroides; es decir, dos municipios fueron considerados vecinos si la distancia entre sus cabeceras municipales resulta ser igual o menor a $54.6 \mathrm{~km}$, lo que representa la distancia máxima existente entre dos cabeceras municipales cualesquiera, del total de 121 cabeceras que conformaron Chiapas al año 2015 (Ord y Getis. 1995).

Una vez definida la estructura de vecindades, se puede construir una matriz binaria de contigüidades de forma que $\delta \mathrm{ij}=1$, si los municipios $\mathrm{i}, \mathrm{j}$ son vecinos, y $\delta \mathrm{ij}=0$ en caso contrario, con lo que la matriz de contigüidades debe ser simétrica, $\delta \mathrm{ij}=\delta \mathrm{ji}$, para todo $\mathrm{i}, \mathrm{j}$ y $\delta \mathrm{ii}=$ 0 por convención (Chasco 2003).

La matriz de contigüidades permite identificar relaciones de vecindad, pero no cuantifica la importancia de las relaciones entre vecinos, por lo que se define una matriz de ponderadores espaciales de elementos (wij), la cual se construye estandarizando los renglones de la matriz de contigüidades, donde el elemento i de cada renglón j es dividido por la suma de los contactos del renglón:

$$
w_{i j}=\frac{\delta_{i j}}{\sum_{j} \delta_{i j}}
$$

De forma que, la suma de los pesos de cada renglón será igual a la unidad, y la suma de todos los renglones de la matriz será igual al total de unidades espaciales (Cliff y Ord 1981).

\section{2 Índice de Moran}

Los índices de autocorrelación espacial tienen la capacidad de resumir en un único número el nivel de dependencia entre la variable y su distribución en el espacio (Anselin, 1995); con lo que permiten detectar agrupaciones o clústeres espaciales de la variable. Uno de los estadísticos más conocidos de autocorrelación espacial es el índice de Moran $^{3}$, que en su versión global cuantifica la distancia de las unidades espaciales $(i, j)$, respecto de su promedio ponderado por la matriz de pesos espaciales wij (Cliff y Ord, 1981). Cuando se utiliza una matriz de pesos espaciales estandarizada por renglones, el estadístico de Moran puede calcularse como:

$$
I=\frac{\sum_{i} \sum_{j} w_{i j}\left(y_{i}-\bar{y}\right)\left(y_{j}-\bar{y}\right)}{\sum_{i}\left(y_{i}-\bar{y}\right)^{2}}
$$

\footnotetext{
${ }^{3}$ El índice de Moran se construye en dos versiones: Moran global y Moran local. La diferencia radica en que el primero es una medida de autocorrelación espacial para todas las regiones analizadas, y el segundo mide el grado de autocorrelación entre una región y sus vecinos. En ambos casos, la correlación se estima considerando la distancia y un atributo dado.
} 


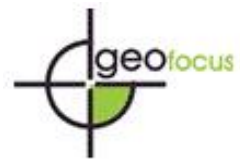

Núñez Medina, G., Jiménez Acevedo, H.M. (2021). Análisis espacial de la fecundidad adolescente y programas sociales en Chiapas. GeoFocus, Revista Internacional de Ciencia y Tecnología de la Información Geográfica, 27, 115-134. http://dx.doi.org/10.21138/GF.557

Es importante señalar que la media teórica del índice de Moran está dada por:

$$
E[I]=\frac{-1}{N-1}
$$

por lo que su valor esperado está en función del tamaño de la muestra $(\mathrm{N})$, que es negativo y tiende asintóticamente a cero conforme aumenta el tamaño de muestra.

El índice de Moran I es un estadístico cuya distribución estandarizada toma la forma de una normal estándar:

$$
z=\frac{I-E[I]}{\sqrt{\operatorname{var}(I)}} \sim N(0,1)
$$

Valores de $\mathrm{z}$ significativamente distintos de cero son evidencia de la presencia de autocorrelación espacial, lo que indica que un p-value inferior a 0.05 , es un indicador para rechazar la hipótesis nula de no autocorrelación espacial.

La tabla 1 muestra los valores del índice de Moran global para las variables analizadas, en general, todas presentan un importante nivel de asociación espacial positiva.

Tabla 1: índice de Moran

\begin{tabular}{|l|r|r|r|}
\hline \multicolumn{1}{|c|}{ Variables } & I - moran & \multicolumn{1}{c|}{$\mathbf{Z}$} & p-value \\
\hline TEF10_19 & 0.134 & 7.419 & 0.00000 \\
\hline Escmedia & 0.171 & 9.371 & 0.00000 \\
\hline Ingresomes & 0.115 & 6.423 & 0.00000 \\
\hline Prospera & 0.060 & 3.573 & 0.00000 \\
\hline segpopular & 0.061 & 3.620 & 0.00000 \\
\hline
\end{tabular}

Fte.: Elaboración propia con datos del INEGI (2015).

En la tabla 1, se observa que los valores del índice de Moran resultaron ser positivos en todos los casos (además presentaron altos niveles de significancia estadística, z mayor a 2 y un $p$ value menor a 0.05 ), lo que indica la presencia de autocorrelación espacial positiva para las variables: fecundidad adolescente (10-19 años), escolaridad media femenina, ingreso familiar medio, proporción de mujeres que recibe ayuda gubernamental y acceso a Seguro Popular. Los resultados de la tabla 1 permiten afirmar que los municipios de Chiapas en 2015, presentan altos niveles de fecundidad adolescente, y que están rodeados a su vez por municipios que alcanzan tasas de fecundidad adolescente por encima del promedio estatal.

Si bien, el índice de Moran global muestra evidencia estadística de la existencia de conglomerados, carece de la capacidad para identificar espacialmente dichos conglomerados, razón por la que deben utilizarse indicadores espaciales locales. 
Núñez Medina, G., Jiménez Acevedo, H.M. (2021). Análisis espacial de la fecundidad adolescente y programas sociales en Chiapas. GeoFocus, Revista Internacional de Ciencia y Tecnología de la Información Geográfica, 27, 115-134. http://dx.doi.org/10.21138/GF.557

\subsection{Modelos de regresión espacial}

Los modelos panel espacial dan cuanta de tres tipos de interacción: (1) interacción endógena espacial de la variable dependiente (y) entre unidades espaciales; (2) interacción exógena entre la variable dependiente $(y)$ y variables independientes $(x)$; (3) interacción entre los términos de error (Elhorst 2014, LeSage y Fischer 2008).

Los métodos de regresión espacial se fundamentan en la generalización de criterios basados en la cercanía tales como la distancia; criterios que permiten estructurar la dependencia espacial, donde la matriz $W$ de pesos espaciales es fundamental en la especificación de los modelos (Getis y Ord 1992).

El modelo saturado de regresión espacial está dado por:

$$
\begin{aligned}
y_{i}=\lambda W y_{i}+X_{i} \beta+W X_{i} \theta+u_{i} \\
u_{i}=\rho W u_{i}+\varepsilon_{i} \\
\varepsilon_{i} \sim N\left(0, \sigma^{2}\right)
\end{aligned}
$$

Wy denota los efectos de interacción endógena con las unidades espaciales, $W X$ representa la matriz de efectos de interacción exógena, $W u$ los efectos de interacción de los términos de error con las unidades espaciales, $\lambda$ es el coeficiente espacial autorregresivo, $\rho$ el coeficiente de errores espaciales, $\theta$ y $\beta$ son los vectores de parámetros a estimar y $W$ es la matriz de pesos espaciales (Green 2008, LeSage 2004).

A partir del modelo saturado de regresión espacial se derivan el modelo espacial autorregresivo (SAR) cuando $\theta=0$ y $\rho=0$; y el modelo de errores espaciales (SEM) cuando $\lambda=0$ y $\theta=0^{4}$. El modelo estructurado de rezago espacial o modelo espacial autorregresivo puede estimarse adicionalmente, suponiendo que no se cumple el supuesto de heterocedasticidad, lo que indicaría que la varianza del error aleatorio es diferente para cada unidad espacial, en cuyo caso debe estimarse el parámetro $\sigma^{2}$ (modelo SAR-ht) de la ecuación (1); el mismo caso se presenta para el modelo SEM-ht. Adicionalmente, se estimó el modelo SARAR conocido como modelo autorregresivo de errores espaciales, el mismo se obtiene al combinar los modelos SAR y SEM, (LeSage y Pace 2009).

Una vez estimados los modelos, las pruebas de especificación para elegir el modelo más adecuado representan un paso fundamental. Algunas pruebas priorizan la existencia de correlación espacial, mientras que otras buscan medir el grado de ajuste del modelo a los datos. En nuestro caso, utilizamos el segundo criterio y optamos por el criterio de información de Akaike (AIC), prueba que proporciona una medida de calidad de ajuste del modelo, en términos de un criterio de selección diseñado a partir de la función de máxima verosimilitud y del número de parámetros estimados. El modelo elegido debe ser el que alcanza el AIC de valor mínimo (Cliff y Ord 1981, Cressie 1993).

\footnotetext{
${ }^{4}$ Existen otros modelos de regresión espacial, tales como el modelo espacial Durbin (SDM) cuando $\lambda=0$ y el modelo espacial autorregresivo y de error espacial (SAC) cuando $\theta=0$.
} 


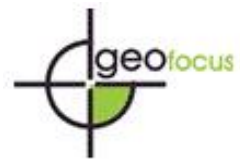

Núñez Medina, G., Jiménez Acevedo, H.M. (2021). Análisis espacial de la fecundidad adolescente y programas sociales en Chiapas. GeoFocus, Revista Internacional de Ciencia y Tecnología de la Información Geográfica, 27, 115-134. http://dx.doi.org/10.21138/GF.557

\section{Resultados}

Las medidas de autocorrelación locales, denominadas también indicadores Locales de Asociación Espacial o LISA, permiten detectar la presencia de conglomerados o clústeres. El diagrama de dispersión de Moran (Anselin 1995) es un indicador LISA, que permite visualizar la correlación lineal de las unidades espaciales en función de la variable analizada y su retardo espacial $^{5}$. El eje X representa las desviaciones respecto de la media de la variable analizada, mientras que en el eje Y se presenta el retardo espacial y la pendiente representa el índice de Moran global.

\section{Figura 2. Diagrama de dispersión de Moran de la fecundidad adolescente 10-19}

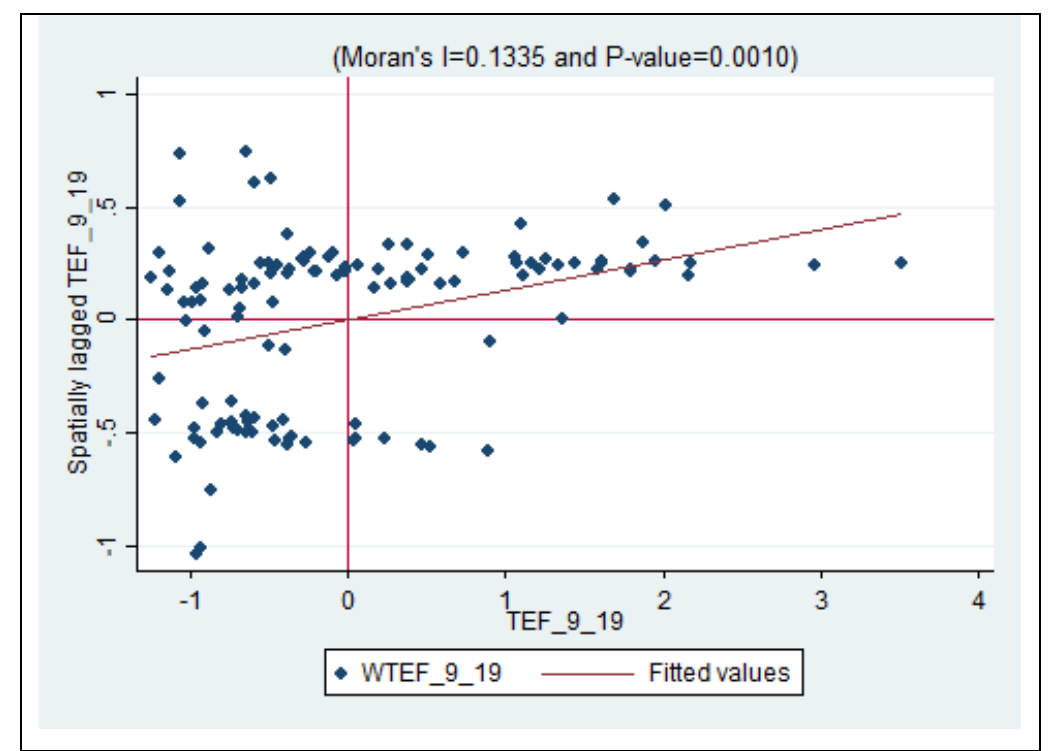

Fte.: Elaboración propia con datos de la encuesta intercensal (INEGI 2015)

La figura 2 muestra el diagrama de dispersión de Moran para la fecundidad adolescente (10-19) en los municipios de Chiapas, al año 2015. Es notoria la inclinación de la recta, lo que confirma los altos niveles de autocorrelación espacial positiva de la variable analizada.

Las gráficas 3(a-d) muestran los diagramas de dispersión de Moran para las variables que se espera influyan sobre la distribución espacial de la fecundidad adolescente en Chiapas. Los diagramas, en todos los casos, presentan una inclinación positiva de la recta de regresión entre la variable y la distribución espacial de la misma, lo que implica una correlación espacial positiva, la cual es especialmente importante para el caso de la escolaridad femenina promedio municipal (figura 3a).

\footnotetext{
${ }^{5}$ El retardo espacial es un concepto que cuantifica la relación que mantiene cada unidad espacial con sus unidades vecinas. Se define en términos del grado de semejanza que alcanzan los valores promedio ponderados de la variable analizada (de la unidad estudiada), respecto de sus unidades vecinas.
} 


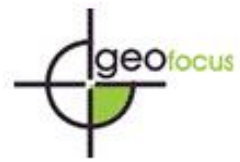

Núñez Medina, G., Jiménez Acevedo, H.M. (2021). Análisis espacial de la fecundidad adolescente y programas sociales en Chiapas. GeoFocus, Revista Internacional de Ciencia y Tecnología de la Información Geográfica, 27, 115-134. http://dx.doi.org/10.21138/GF.557

\section{Figura 3. Diagrama de dispersión de Moran}

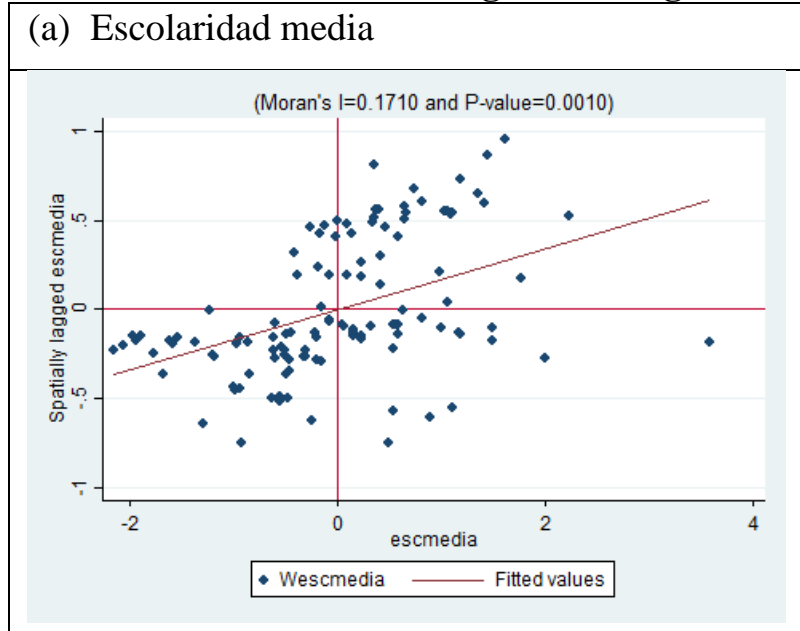

(b) Ingreso medio mensual
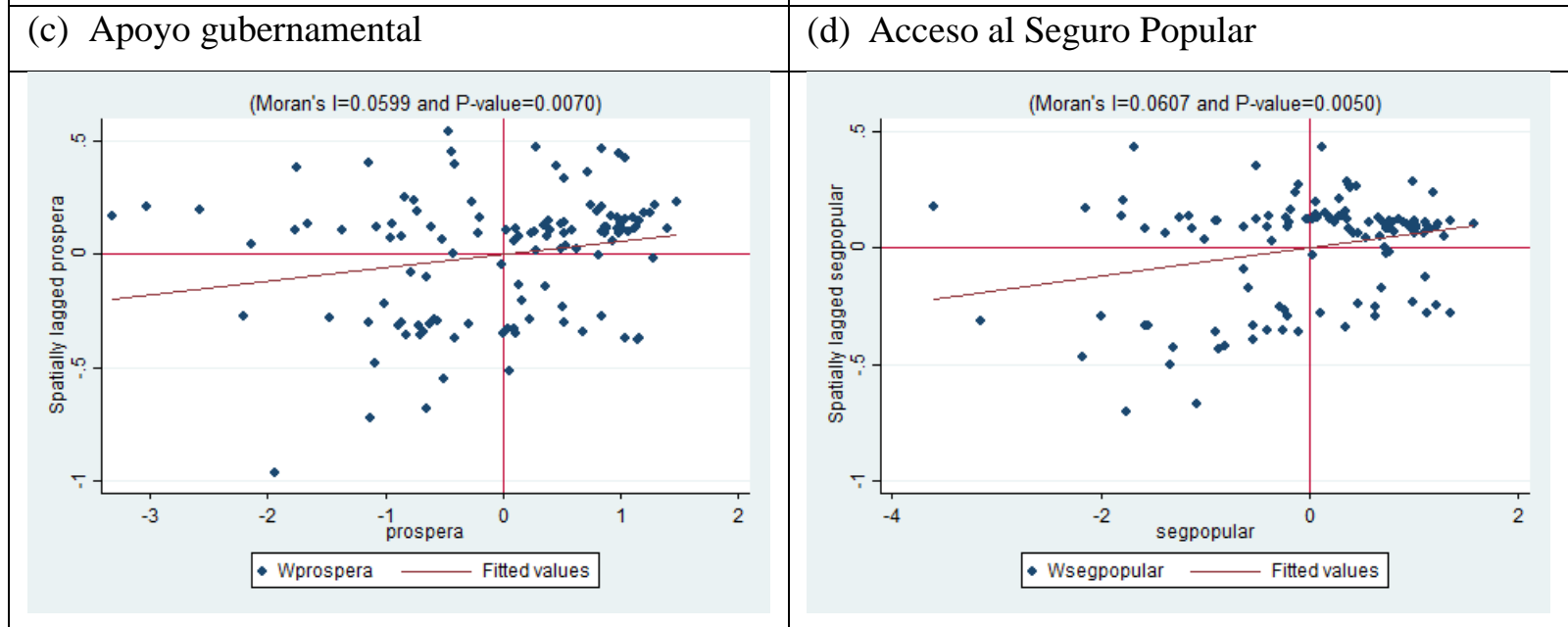

Fte.: Elaboración propia con datos de la encuesta intercensal (INEGI 2015).

La formación de conglomerados puede verse en los mapas 1 y 2 para la tasa de fecundidad adolescente, y para cada una de las variables analizadas. Los conglomerados se forman al agrupar los municipios según los cuadrantes formados en el diagrama de Moran. De modo que, en el mapa 1 se identifican los clústeres, que agrupan municipios con distintas combinaciones de valores altos o bajos y sus respectivas combinaciones, a partir de lo cual se identifican cuatro tipos de conglomerados:

1) High-High: unidades territoriales con valores por encima del promedio rodeadas por unidades con valores superiores a la media, llamados conglomerados calientes o hot spots, y que se representan de color rojo;

2) Low-Low: conglomerados formados por unidades espaciales cuyo valor de análisis es inferior al promedio, que están rodeados por unidades con valores por bajo la media, son llamados conglomerados fríos o cold spots, y que en general se dibujan de color azul;

3) Low-High: son conglomerados formados por unidades espaciales con valores por debajo de la media, rodeados por unidades con valores sobre la media, y que se dibujan color naranja;

4) High-Low: presenta conglomerados de unidades espaciales con valores por encima del promedio, rodeados por unidades espaciales que presentan valores por debajo de la media, y que suelen colorearse en celeste; 


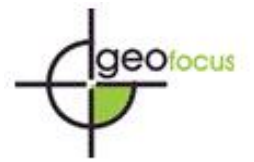

Núñez Medina, G., Jiménez Acevedo, H.M. (2021). Análisis espacial de la fecundidad adolescente y programas sociales en Chiapas. GeoFocus, Revista Internacional de Ciencia y Tecnología de la Información Geográfica, 27, 115-134. http://dx.doi.org/10.21138/GF.557

5) No Significativo: son las unidades espaciales que no presentan niveles de correlación significativa entre unidades vecinas, con relación a la variable analizada (LeSage y Pace 2009, Elhorst 2014).

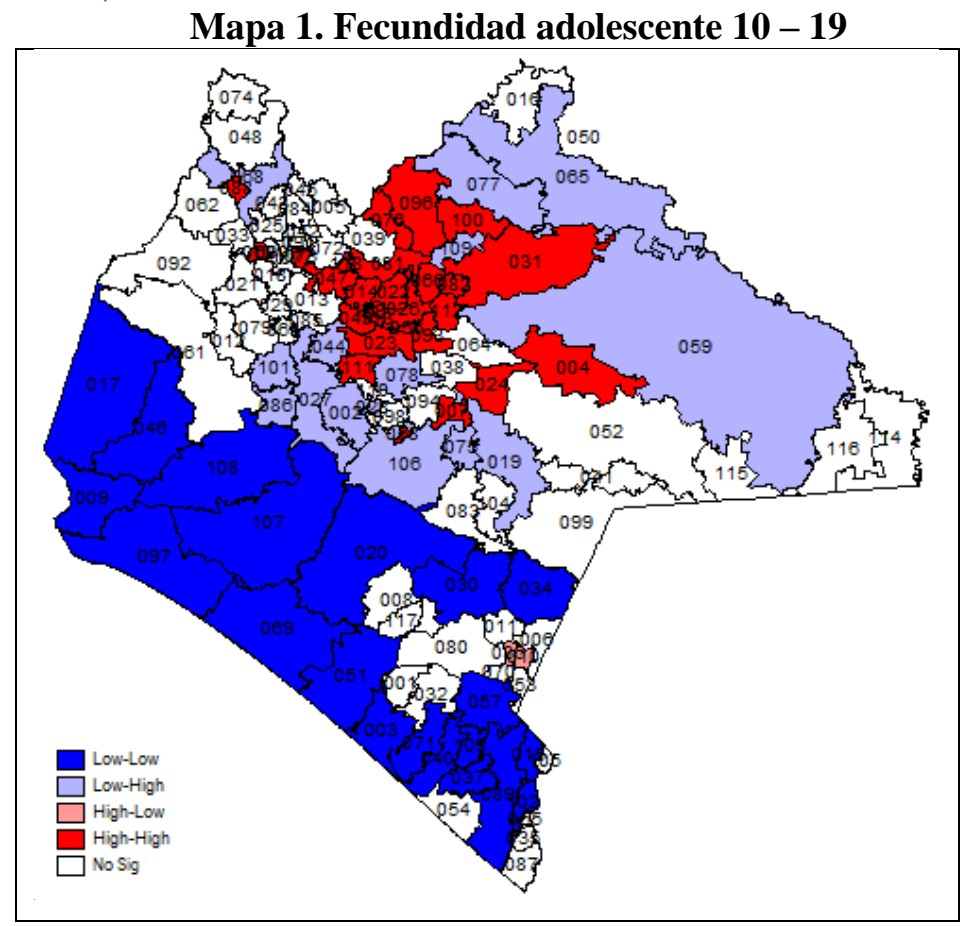

Fte.: Elaboración propia.

El mapa 1 muestra el comportamiento espacial de la fecundidad adolescente (10-19) en los municipios de Chiapas, al año 2015. En concordancia con la clasificación realizada por el índice de Moran local, los municipios en blanco representan unidades espaciales con niveles de correlación estadística no significativa.

El mapa 1 de autocorrelación espacial de la fecundidad adolescente permite identificar dos conglomerados calientes (rojos), es decir, dos conjuntos de municipios con altos niveles de fecundidad adolescente rodeados de municipios con niveles de fecundidad adolescente por encima de la media estatal. Un primer conglomerado se encuentra ubicado en la zona Norte y Altos de Chiapas, que comprende los municipios de Chalchihuitán, Tila, Sabanilla y Chenalhó, entre otros; el segundo conglomerado abarca los municipios de Altamirano, Chanal y Amatenango del Valle. Además se distingue un conglomerado frío (azul) de municipios donde se observan niveles relativamente bajos de fecundidad adolescente, que están rodeados de municipios con niveles de fecundidad adolescente que presentan niveles por debajo del promedio estatal, los cuales se encuentran ubicados en la zona Itsmo-Costa y la zona del Soconusco, que comprende los municipios de Arriaga, Tonalá, Villaflores, Jiquipilas, Tapachula, Mazatán y Huehuetán, entre otros.

El mapa 2a hace referencia a la distribución espacial de la escolaridad media municipal de las mujeres chiapanecas. En el mismo se pueden distinguir dos conglomerados de color rojo, uno en la zona Itsmo-Costa, que comprende los municipios de Arriaga, Tonalá, Villaflores, y Jiquipilas entre otros; y un segundo conglomerado en la zona del Soconusco, que abarca los municipios de Tapachula, Mazatán y Huehuetán, entre otros (ver mapa 2a). Adicionalmente, en el mapa puede apreciarse un gran conglomerado frio, ubicado en la zona Norte del estado, en municipios como Chalchihuitán, Tila, Sabanilla y Chenalhó, entre otros, conglomerado que representa la zona con 


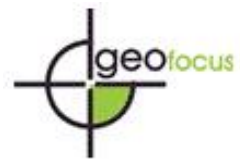

Núñez Medina, G., Jiménez Acevedo, H.M. (2021). Análisis espacial de la fecundidad adolescente y programas sociales en Chiapas. GeoFocus, Revista Internacional de Ciencia y Tecnología de la Información Geográfica, 27, 115-134. http://dx.doi.org/10.21138/GF.557

los menores niveles de escolaridad femenina en el estado. Los mapas 2(b-d) presentan un análisis de la distribución espacial de los determinantes que se consideraron más importantes para entender el comportamiento espacial de la fecundidad adolescente en el estado de Chiapas, a saber: el ingreso promedio mensual familiar, la proporción de hogares que recibe apoyo gubernamental y el porcentaje de mujeres que se encuentran inscritas en el Seguro Popular; el comportamiento de los mismos es similar al de la escolaridad media femenina municipal, por lo que se omite su descripción.

\section{Mapa 2: Autocorrelación espacial de la fecundidad en Chiapas}

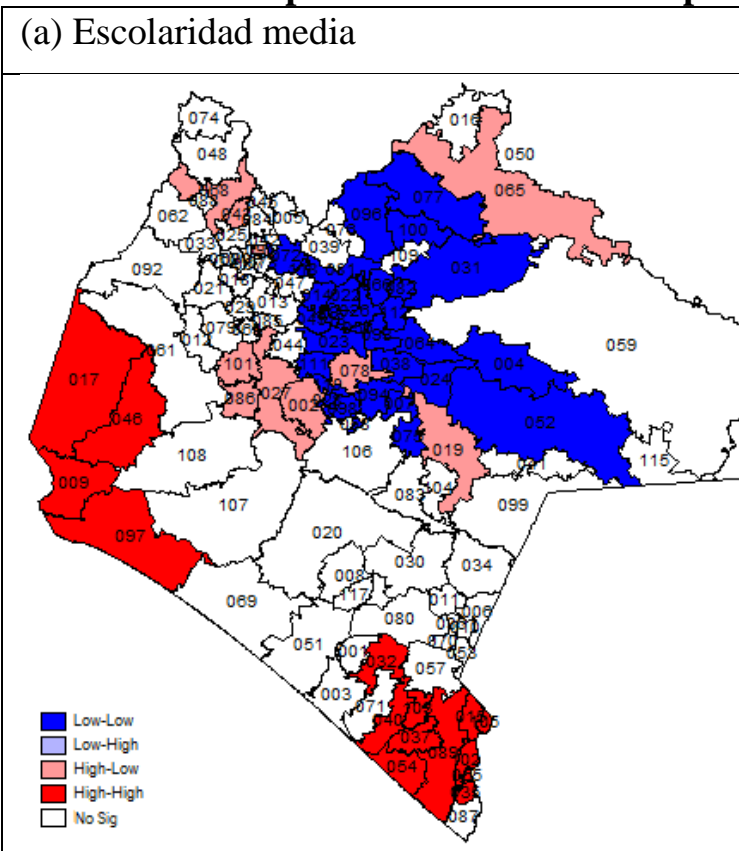

(b) Ingreso medio mensual

\section{(c) Apoyo gubernamental}
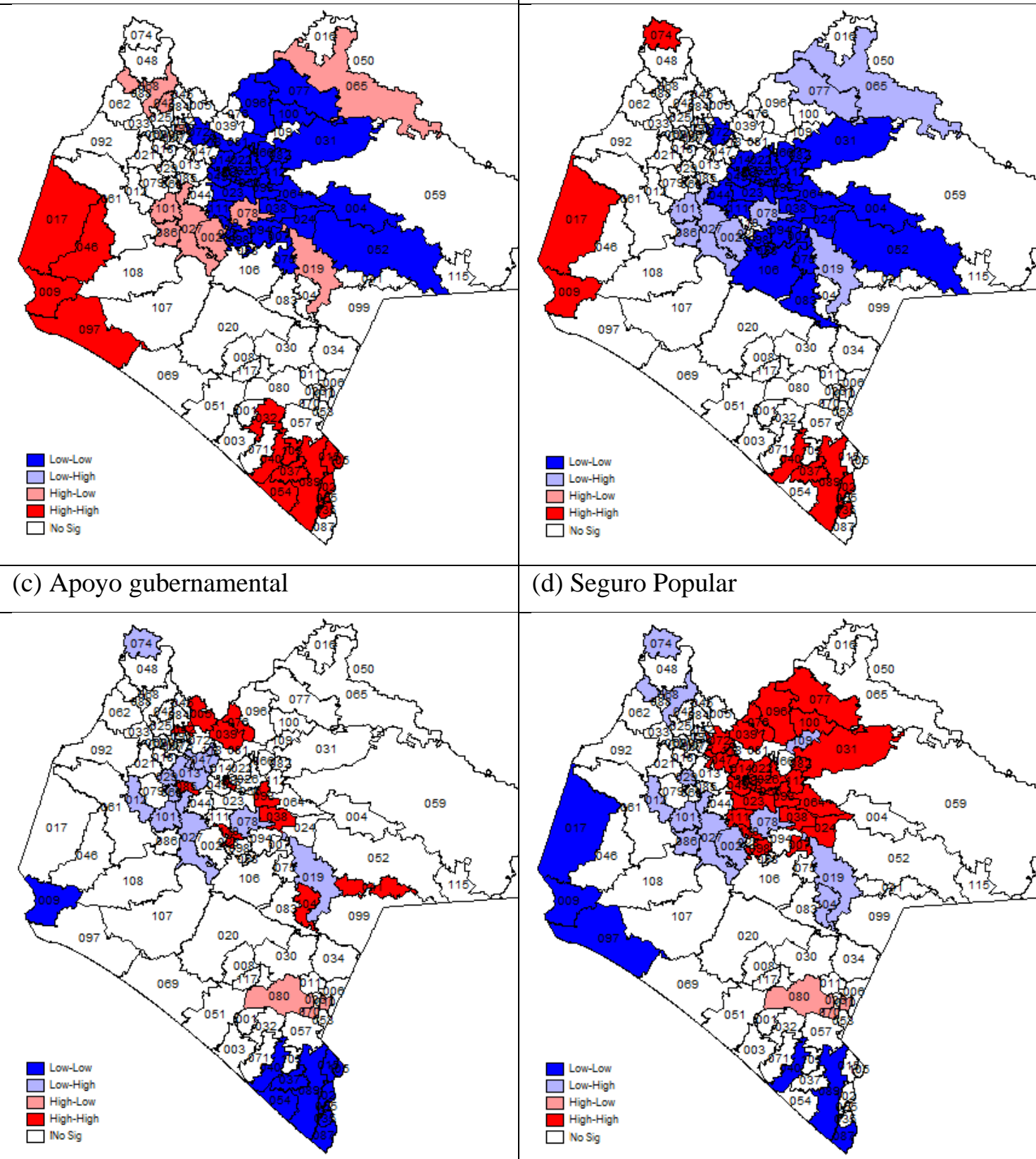

(d) Seguro Popular

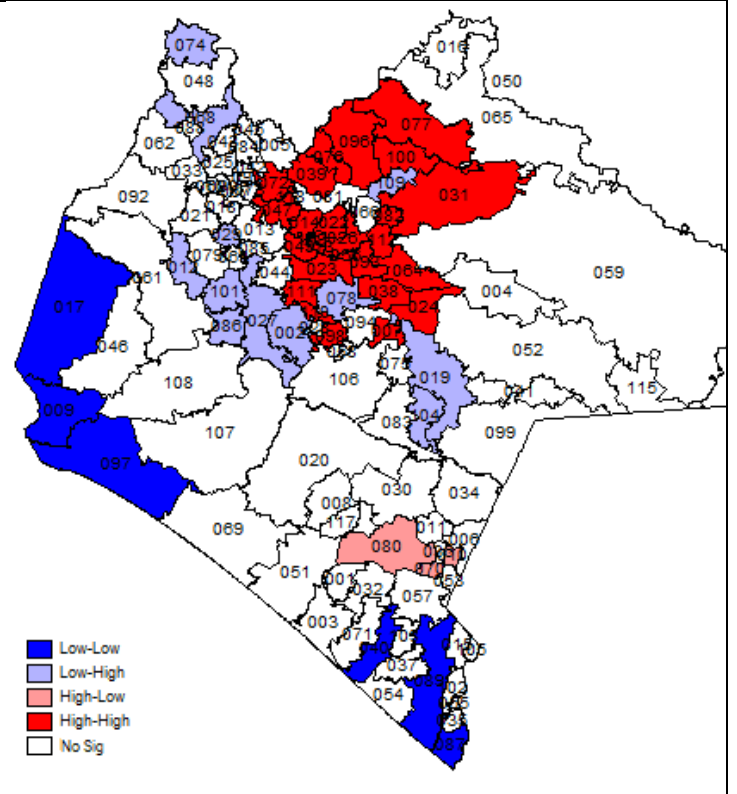

Fte.: Elaboración propia con datos de la encuesta intercensal (INEGI 2015). 
Núñez Medina, G., Jiménez Acevedo, H.M. (2021). Análisis espacial de la fecundidad adolescente y programas sociales en Chiapas. GeoFocus, Revista Internacional de Ciencia y Tecnología de la Información Geográfica, 27, 115-134. http://dx.doi.org/10.21138/GF.557

Una vez presentado el análisis exploratorio de datos espaciales, se procede a modelar la heterogeneidad espacial de la fecundidad adolescente en los municipios de Chiapas, para lo que se ajustaron seis modelos: un modelo de regresión lineal simple, 2 modelos de rezago espacial, 2 modelos de error espacial y un modelo mixto. El modelo de regresión lineal simple fue ajustado con fines de comparación. Los resultados de las estimaciones realizadas a la totalidad de los modelos pueden verse en la tabla 2.

Tabla 2: Modelos panel espacial

\begin{tabular}{|c|c|c|c|c|c|c|}
\hline TEF10_19 & LM & SAR & SAR-ht & SEM & SEM-ht & SARAR \\
\hline escmedia & $-0.0505 * *$ & $-0.02799 *$ & $-0.03118 * *$ & $-0.0333 * *$ & $-0.03302 * *$ & $-0.03202 \quad * *$ \\
\hline ingresomes & $0.0000 * *$ & -0.00000 & -0.00001 & -0.0001 & -0.00001 & -0.00001 \\
\hline prospera & $0.0000 * * *$ & $0.27433 * * *$ & $0.26853 * *$ & $0.2429 * *$ & $0.24225 * *$ & $0.25080 \quad * *$ \\
\hline segpopular & $-0.1126 *$ & $-0.17866 * *$ & $-0.17631 * *$ & $-0.1636 * *$ & $-0.16319 *$ & $-0.16660 \quad *$ \\
\hline Cons & $0.5190 *$ & 0.17742 & $0.20744 *$ & $0.2699 * *$ & $0.26923 * *$ & $0.23954 \quad *$ \\
\hline lambda & & $0.43895 * *$ & $0.31592 *$ & & & 0.16927 \\
\hline Rho & & & & 0.5185 & $0.53427 *$ & 0.36900 \\
\hline sigma2 & & & $0.00328 * * *$ & & $0.00325 * * *$ & $0.00326 * * *$ \\
\hline AIC & -321.377. & -323.483 & -323.370 & -323.396 & -323.689 & -323.956 \\
\hline
\end{tabular}

Fte.: Elaboración propia con datos de la encuesta intercensal (INEGI 2015).

El modelo SAR utiliza un vector de rezago espacial para modelar los efectos que las covariables ejercen sobre los niveles de fecundidad adolescente municipal. El parámetro $\lambda$ refleja la fuerza de la dependencia espacial, si $\lambda$ es estadísticamente igual a cero, el modelo SAR se transforma en un modelo de regresión lineal simple (LM). Puede observarse en la tabla 2 que el parámetro $\lambda$ resultó ser estadísticamente distinto de cero, por lo que SAR resulta ser un modelo válido.

El modelo SAR-ht debe estimarse cuando se sospecha de la presencia de heterocedasticidad en el modelo de autocorrelación. En este tipo de modelos $\sigma^{2}$ busca capturar estructuralmente la varianza de los residuos, bajo el supuesto de que la misma es no constante entre unidades espaciales. La estimación para la varianza resulto ser significativamente distinta de cero, con lo que se reafirma la importancia del modelo.

El modelo SEM es esencialmente un modelo lineal generalizado con errores espacialmente autocorrelacionados, por lo que no supone explícitamente retrasos espaciales de la variable dependiente, de modo que los parámetros $\beta$ estimados pueden interpretarse en el mismo sentido que se hace en un modelo de regresión lineal. Puede observarse en la tabla 2 que el parámetro de errores espaciales $\rho$ resultó no significativo, con lo que el modelo SEM se reduce a un modelo LM.

El modelo SEM-ht de errores espaciales con heterogeneidad analiza la interacción entre unidades espaciales restringiéndola a los términos de error, siempre que la varianza entre unidades espaciales no sea constante, en caso contrario, se supondrá que el número de vecinos es constante para cada unidad espacial (con lo que la diagonal de la matriz de varianzacovarianza tendrá un número constante de elementos), por lo que se tendrá un modelo homoscedástico. El parámetro autorregresivo $\rho$ modela la autocorrelación de los errores. La correlación disminuye según el orden de vecindad, dado que la matriz $\mathrm{W}$ representa un patrón 


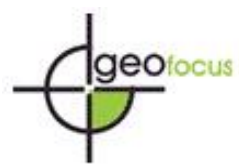

Núñez Medina, G., Jiménez Acevedo, H.M. (2021). Análisis espacial de la fecundidad adolescente y programas sociales en Chiapas. GeoFocus, Revista Internacional de Ciencia y Tecnología de la Información Geográfica, 27, 115-134. http://dx.doi.org/10.21138/GF.557

global de autocorrelación espacial. Como puede verse en la tabla 2, el parámetro de error espacial $\rho$ resultó ser significativo, al igual que la varianza de los errores espaciales.

La especificación del modelo SARAR incorpora un proceso autorregresivo de dependencia espacial, al tiempo que integra un proceso autorregresivo del error espacial (Tiefelsdorf 2000), de forma que se estima tanto $\lambda$ como $\rho$. El parámetro $\lambda$ y el parámetro $\rho$ resultaron ser estadísticamente no significativos, por lo que el modelo debe ser descartado, debido a que no captura adecuadamente los efectos espaciales autorregresivos (Griffith 2000).

Para la selección del modelo que mejor ajusta los datos espaciales se utilizó el criterio AIC. Es importante señalar que, la prueba no proporcionó información concluyente para diferenciar entre modelos, debido a que los resultados son muy similares entre los modelos espaciales ajustados. Sin embargo, se seleccionó el modelo SEM-ht debido a que presenta el menor AIC después del modelo SARAR, que resultó ser no significativo. El modelo SEM-ht tiene una buena consistencia en cuanto al signo y magnitud de los parámetros estimados, el valor del parámetro $\rho$ de error espacial alcanzó un buen nivel de significancia con lo que se obtuvo un modelo parsimonioso, que captura los posibles efectos generados por la heterogeneidad de los errores no observables entre las unidades espaciales analizadas.

Los coeficientes del modelo SEM-ht se interpretan, como hemos mencionado, en el mismo sentido que un modelo de regresión lineal, donde el coeficiente de regresión $\beta$ expresa el cambio en el número de unidades que experimentaría la tasa de fecundidad adolescente por cada unidad que aumente la variable independiente. Lo que significa que un incremento de un año en la escolaridad femenina promedio, impactaría en una reducción de 0.033 puntos porcentuales de la tasa de fecundidad adolescente municipal. La incorporación de las adolescentes al Seguro Popular tiene efectos positivos sobre la reducción de los embarazos en adolescentes, donde el incremento de un punto porcentual en la afiliación al Seguro Popular tendría un impacto de 0.16 puntos porcentuales en la reducción de la tasa de fecundidad adolescente.

Se debe señalar que, el efecto más importante es el ejercido por los subsidios gubernamentales, donde el incremento de un punto en el porcentaje de hogares apoyados a través de programas del gobierno, implicaría un incremento de 0.24 puntos porcentuales sobre la tasa de fecundidad adolescente municipal; efecto que no se equipara al asociado al ingreso laboral familiar, cuyo impacto sobre la fecundidad adolescente es estadísticamente inexistente, lo que implica entre otras cosas, que no es la variable ingreso la que afecta los niveles de fecundidad de las adolescentes, sino que son los ingresos obtenidos vía subsidios los que incentivan la natalidad entre las adolescentes.

\section{CONCLUSIONES}

El principal objetivo ha sido entender el efecto que los programas de apoyo gubernamental han ejercido sobre los niveles de fecundidad adolescente en los municipios de Chiapas, para cumplir con dicho objetivo, se utilizaron variables que permitieron modelar la relación entre la fecundidad adolescente y la educación de las mujeres, el ingreso familiar laboral y el acceso a servicios médicos (medido a través de la incorporación al Seguro Popular). A partir de ello, se planteó una estrategia de modelación con base en una metodología de regresión espacial, con la finalidad de analizar la heterogeneidad observada entre los municipios con relación a los niveles de fecundidad adolescente observados al año 2015.

Es importante destacar que, durante la fase de análisis exploratorio de datos espaciales, todas las variables presentaron niveles razonablemente buenos de autocorrelación espacial, con lo que era 


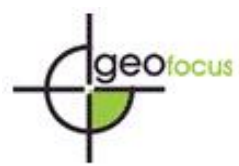

Núñez Medina, G., Jiménez Acevedo, H.M. (2021). Análisis espacial de la fecundidad adolescente y programas sociales en Chiapas. GeoFocus, Revista Internacional de Ciencia y Tecnología de la Información Geográfica, 27, 115-134. http://dx.doi.org/10.21138/GF.557

de esperarse que se obtendría un buen nivel de ajuste de los modelos de regresión espacial con relación a los niveles observados de fecundidad adolescente. Lo cual ocurrió en todos los casos y para todas las variables, salvo para el ingreso familiar, variable que parece no ejercer ningún efecto (espacial y no espacial) sobre los niveles de fecundidad en adolescentes.

Al comparar los modelos de rezago espacial y de error espacial, se pudo ver que los modelos de error espacial presentaron un mejor grado de ajuste respecto de los datos (un menor AIC). También fue notoria la presencia de heterocedasticidad en todos los modeles, lo que implica la existencia de un efecto espacial no observado, y por tanto, no capturado por las covariables utilizadas durante esta investigación. Efecto que ejerce una importante influencia sobre el nivel observado de fecundidad adolescente en los municipios de Chiapas, y que fue captado a través del término de error.

Se puede concluir que, a pesar de que el ingreso laboral no haya tenido efecto espacial directo sobre el comportamiento de la fecundidad adolescente, el papel de la escolaridad femenina promedio y la cobertura de los servicios de salud municipales (Seguro Popular) lograron capturar una parte importante de la variabilidad de la fecundidad entre municipios, por lo que, dichas variables resultan ser fundamentales para incidir de forma efectiva en la reducción de embarazos en adolescentes en Chiapas. Es importante señalar que, los resultados muestran que el ingreso medio familiar no ejerce efecto alguno sobre los niveles de fecundidad adolescente; mientras que los ingresos provenientes de apoyos gubernamentales, que son generalmente entregados de manera directa a las mujeres madres de familia, sí presentaron un efecto espacial positivo, incrementando de forma importante los niveles de fecundidad de las adolescentes en Chiapas, situación que se presenta con mayor fuerza en los municipios con menores niveles de escolaridad.

Los resultados presentados son producto de la aplicación empírica de una serie de modelos econométricos, cuyo objetivo es mostrar la estimación del efecto que una serie de variables ejercen sobre el comportamiento espacial de la tasa de fecundidad adolescente, lo que incluye la detección de conglomerados con altos y bajos niveles de fecundidad. La estimación de los modelos se realizó utilizando el paquete estadístico R. Es importante señalar que, la investigación presentada es un estudio de cohorte transversal acotado al año 2015 y a los 121 municipios que integran el Estado de Chiapas, y que en ningún caso se pretende generalizar o extrapolar los resultados aquí presentados, sino que por el contrario, los resultados revelan la necesidad de realizar estudios enfocados específicamente a un análisis más profundo de los determinantes que intermedian entre el efecto de los subsidios gubernamentales y el incremento de la fecundidad en adolescentes en Chiapas, dado que la relación en principio parece ser perversa; sin embargo, es necesario considerar que los subsidios otorgados a las madres de familia (incluidas madres adolescentes) tienen múltiples propósitos, y son ejercidos para solventar un gran número de carencias y necesidades presentes en los municipios estudiados.

\section{Referencias bibliográficas}

Anselin, L. (1988). Spatial econometrics: Methods and Models. Boston, Kluwer Academic Publishers.

Anselin, L. (1995). "Local Indicators of Spatial Associations-LISA", Geographical Analysis, 27, 93-115.

Azevedo, J. P. et al. (2012). Embarazo adolescente y oportunidades en América Latina y el Caribe. Sobre la maternidad temprana, pobreza y logros económicos. Washington, Banco Mundial. 


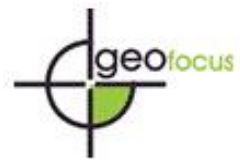

Núñez Medina, G., Jiménez Acevedo, H.M. (2021). Análisis espacial de la fecundidad adolescente y programas sociales en Chiapas. GeoFocus, Revista Internacional de Ciencia y Tecnología de la Información Geográfica, 27, 115-134. http://dx.doi.org/10.21138/GF.557

BM (Banco de México). (2012). Poverty and Labor Brief: The Effect of Women's Economic Power in Latin America and Caribbean. Washington, Banco Mundial.

Berthelon, M. y Kruger, D. (2011). "Risky behavior among youth: Incapacitation effects of school on adolescent motherhood and crime in Chile", Journal of Public Economics, 95, 41-53.

Cliff, A. D. y Ord, J. K. (1981). Spatial processes: Models and Applications. London, Pion Limited.

Cortés D. et al. (2011). "On the design of education Conditional Cash Transfer programs and non-education outcomes: The case of teenage pregnancy", CESifo Working Paper, 3531.

CONAPO (Consejo Nacional de Población). (2010). Dinámica demográfica 1990-2010 y proyecciones de población 2010-2030. http://www.conapo.gob.mx/work/models/CONAPO/ Proyecciones/Cuadernos/07_Cuadernillo_Chiapas.pdf (consultado 15-10-2016).

Cressie, N. (1993). Statistics for spatial data. New York, John Wiley.

Chasco, C. (2003). Econometría espacial aplicada a la predicción-extrapolación de datos microterritoriales. Madrid, Consejería de Economía e Innovación Tecnológica, Comunidad de Madrid.

Ehrenfeld, N. (2011). "Sexualidad, género y violencias: encuentros y desencuentros" en Ehrenfeld, N. (Comp.). Mujeres y acciones: aspectos de género en escenarios diversos. México: Universidad Autónoma Metropolitana-Universidad de Colima, 13-30.

Elhorst, J. P. (2010). "Spatial Panel Data Models" en Fischer, M. y Getis, A. (Eds.). Handbook of Applied Spatial Analysis: Software Tools, Methods and Applications. Berlin, Springer, 377407.

Elhorst, J. P. (2014). Spatial Econometrics, from cross-sectional data to spatial panels. Berlin, Springer.

ENADID. Estrategia Nacional para la Prevención del Embarazo Adolescente. (2016). Gobierno Federal, https://supervisionv030.files.wordpress.com/2016/04/enapea.pdf (consultado 28-10-2016).

ENSANUT. Encuesta Nacional de Salud y Nutrición. (2012). "Instituto Nacional de Salud Pública", http://ensanut.insp.mx/doctos/ENSANUT2012_Sint_Ejec-24oct.pdf (consultado 1510-2016).

Fischer, M. y Getis, A. (Eds.). (2010). Handbook of Applied Spatial Analysis. Software Tools, Methods and Applications. Berlin, Springer.

Flórez Nieto, C. E. et al. (2004). "Fecundidad adolescente en Colombia: incidencia, tendencias y determinantes. Un enfoque de historia de vida". Documento CEDE, 31. Bogotá, Universidad de Los Andes.

Getis, A. y Ord J. K. (1992). "The analysis of spatial association by use of distance statistics", Geographical Analysis, 24, 189-199.

Green W. H. (2008). Econometric analysis. New Jersey, Pearson.

Griffith D. A. (2000). "A linear regression solution to the spatial autocorrelation problem", $J$ Geogr Syst, 2, 2, 141-156.

Guzmán, J. M. et al. (2000). Diagnóstico sobre la salud reproductiva de jóvenes y adolescentes en América Latina y el Caribe. México, EAT. 


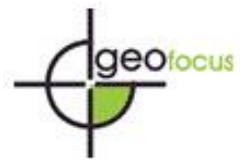

Núñez Medina, G., Jiménez Acevedo, H.M. (2021). Análisis espacial de la fecundidad adolescente y programas sociales en Chiapas. GeoFocus, Revista Internacional de Ciencia y Tecnología de la Información Geográfica, 27, 115-134. http://dx.doi.org/10.21138/GF.557

Hernández, M.F., De la Cruz Muradás, M. y Sánchez, M. (2015). "La situación geográfica de México 2015" en Consejo Nacional de Población. (Coord.). Panorama de la salud sexual y reproductiva. México, CONAPO, 43-71.

INEGI. Instituto Nacional de Estadística y Geografía. (2010). Censo de población y vivienda 2010. México, INEGI.

INEGI. (2015). Encuesta Intercensal 2015. México, INEGI.

Issler, J. R. (2013). "Embarazo en la adolescencia", Revista de Posgrado de la Cátedra VIa Medicina, 107, 11-23. https://med.unne.edu.ar/revistas/revista107/emb_adolescencia.html (consultado 13-10-2016).

LeSage, J. P. (2004). "A family of geographically weighted regression models" en Anselin, L. Florax, R. J. y Rey S. J. (Eds.). Advances in spatial econometrics. Methodology, tools and applications. Berlin: Springer, 241-264.

LeSage, J. P. y Fischer M. M. (2008). "Spatial growth regressions: model specification, estimation and interpretation", Spat Econ Anal 3, 3, 275-304.

LeSage, J. P. y Pace R. K. (2009). Introduction to spatial econometrics. Boca Raton: CRC Press Taylor \& Francis Group.

LPDNNA. Ley para la Protección de los Derechos de Niñas, Niños y Adolescentes. (2000). Cámara de Diputados del H. Congreso de la Unión. DOF: 29-05-2000.

Naciones Unidas. (2003). World Population Prospects: The 2002 Revision, 35-152.

Núñez, J, (2009). "Incidencia del gasto público social en la distribución del ingreso, la pobreza y la indigencia", Archivos de Economía, 359.

Ordóñez, M. y Murad, R. (2002). Variables predictoras de la salud general y de la salud sexual y reproductiva de las mujeres en edad fértil. Estudio a profundidad de la ENDE 2000. Bogotá, Profamilia - Fondo de Población de Naciones Unidas.

Ord, J. K. y Getis, A. (1995). "Local spatial autocorrelation statistics: distributional issues and an application", Geographical Analysis, 27, 286-296.

PROMAJOVEN. Programa de Becas de Apoyo a la Educación Básica de Madres Jóvenes y Jóvenes Embarazadas. (2012). Embarazo adolescente y madres jóvenes en México. Una visión desde el PROMAJOVEN. http://www.promajoven.sep.gob.mx/files/materiales/Embarazo_ Adolescente.pdf

$\mathrm{R}$ Core Team. (2013). $R$ : A language and environment for statistical computing. Vienna, $\mathrm{R}$ Foundation for Statistical Computing. http://www.R-project.org/.

Ripley, B. D. (1981). Spatial Statistics. New York, Wiley.

Stern, C., y Menkes C. (2008). "Embarazo adolescente y estratificación social" en Lerner, S. y Szasz, I. (Coords.). Salud reproductiva y condiciones de vida en México, tomo I. México, El Colegio de México.

Tiefelsdorf, M. (2000). Modelling Spatial Processes. Berlin, Springer-Verlag.

Tobler, W. R. (1970). "A computer movie simulating urban growth in the Detroit region", Economic Geography, 46, 234-240. 


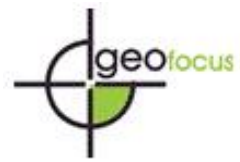

Núñez Medina, G., Jiménez Acevedo, H.M. (2021). Análisis espacial de la fecundidad adolescente y programas sociales en Chiapas. GeoFocus, Revista Internacional de Ciencia y Tecnología de la Información Geográfica, 27, 115-134. http://dx.doi.org/10.21138/GF.557

UNFPA. s/f: "Direct estimation of fertility from survey data containing birth histories", http://demographicestimation.iussp.org/content/direct-estimation-fertility-survey-datacontaining-birth-histories (consultado 14-09-2016)

Valencia, J. y Schiavon, R. (2014). “Adolescentes invisibles: Embarazo en mujeres menores de 15 años en México" en Gobierno Federal: Estrategia Nacional para la Prevención del Embarazo Adolescente. https://supervisionv030.files.wordpress.com/2016/04/enapea.pdf (consultado 28-10-2016).

Welti, C. s/f: "La fecundidad adolescente: implicaciones del inicio temprano de la maternidad", http://www.dgespe.sep.gob.mx/public/genero/PDF/LECTURAS/fecundidad_adolescente.pdf (consultado 16-08-2016).

Welti, C. (2000). "Análisis demográfico de la fecundidad adolescente en México", Papeles de Población, 26. http://www.redalyc.org/pdf/112/11202603.pdf 